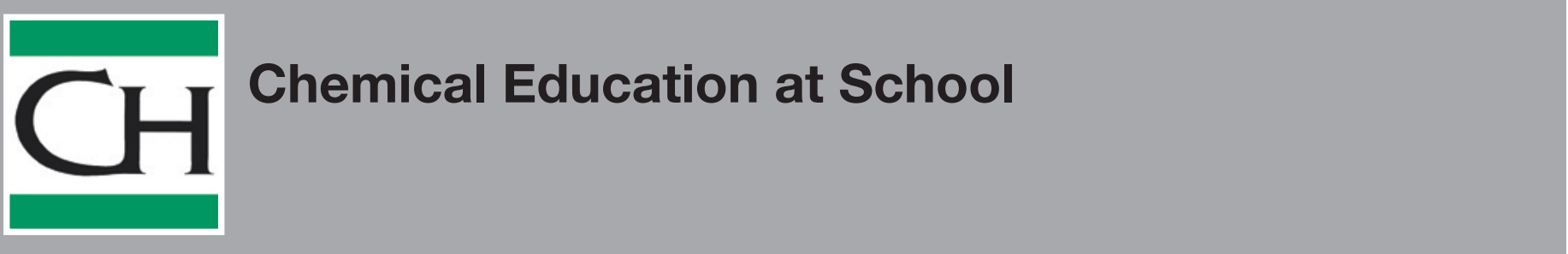

\section{CHI CD \\ A Multimedia Approach to High School Chemistry}

\section{Christian Ammann*}

${ }^{*}$ Correspondence: * Dr. C. Ammann, Mathematisch-Naturwissenschaftliches Gymnasium Rämibühl, Rämistrasse 54, CH-8001 Zürich

E-mail: ammannc@mng.ch

Teaching chemistry is a wonderful business. There are many fascinating experiments leading to many different kinds of teaching and possibilities to make the lessons interesting and captivating for curious pupils.

Several different chemistry text books were and are available for high school chemistry students in Switzerland but, in the author's opinion, none of them could convince as being interesting enough for young people. In 2001 therefore an attempt was initiated to create a new kind of support in learning chemistry. Together with two former pupils, ' $C H I C D$ ', an interactive and multimedia approach to chemistry at high school level was developed.

Computers have been used in most aspects of education. The goal with $C H I C D$ was to create a new kind of 'textbook' that met the demands of our time and desire to have one's computer always at one's disposal. Of course we took into account the advantages of the computer medium and used them to make it easy and exciting to travel through chemistry.

Once installed, $C H I C D$ appears on the screen as an electronic textbook. The content is presented in a comprehensible way and integrated with many pictures to illustrate the text. More than fifty animations are included that demonstrate, for example, the intramolecular reformation of a glucose molecule or the emission of an alpha particle from an instable nucleus. The text can be fol-

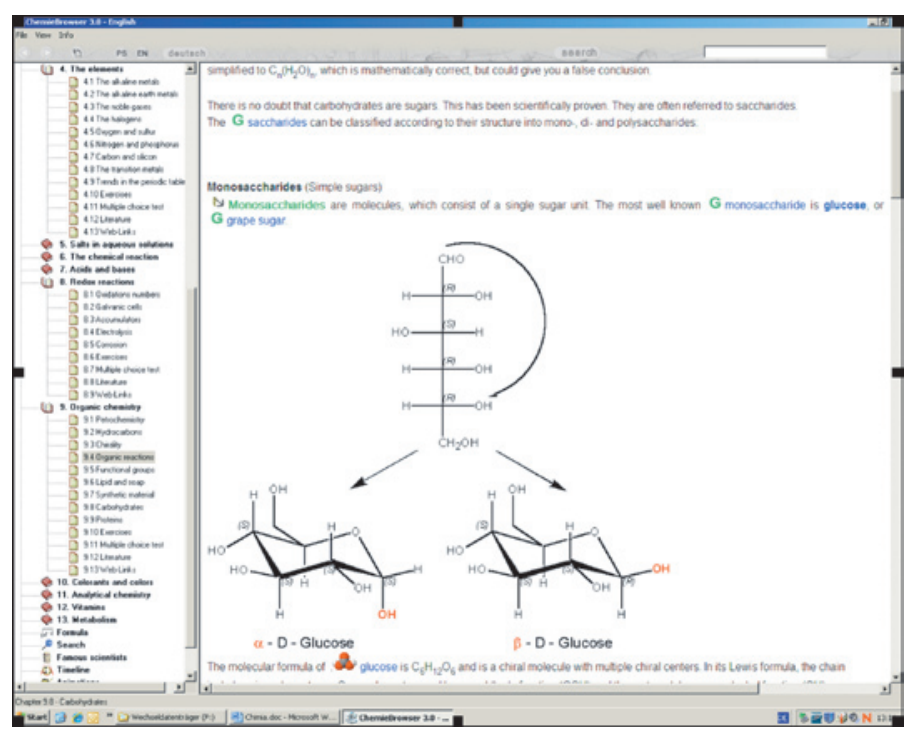

lowed sequentially or related subjects can be accessed directly by the links included. You are referred to even more information by links to web pages. Exercises are provided throughout and a multiple choice test at the end of each chapter will give the student an idea whether his or her preparation is adequate for an upcoming exam. The glossary contains more than two hundred expressions with short explanations and links to texts where more information and background can be obtained. A gallery of important chemists and another with the Nobel Prize laureates is added, linked with the main text. Finally, a time line illustrates progress in chemistry over the years.

Two extra programmes are added that can be used either together with $C H I C D$ or separately. Chemsketch is a powerful tool for drawing molecular structures. Different links in the text open three-dimensional and freely rotatable structures of selected molecules. Another programme, Rasmol, is a protein viewer. Either the desired protein can be accessed from the linked data bank or one of over one hundred protein structures in $C H I C D$ can be viewed.

At this time the third release of $C H I C D$ is available in German, French and English. It is possible to switch easily between the different languages. The fourth release is in preparation and should appear next year (2010). CHI CD is one of the first multimedia chemistry tools for high school level with all the advantages this kind of medium has to offer. Feedback from students has been very good; it seems they like this kind of 'school book' - which is not surprising since this is the generation of users of modern media like computers, mobile phones, mp3 players and so on.

CHI CD is presented on the website www.lerncd.ch where a trial version can be downloaded. A single user $\mathrm{CD}$ is available for SFr. 44.-; orders for classes are discounted.

Received: April 6, 2009

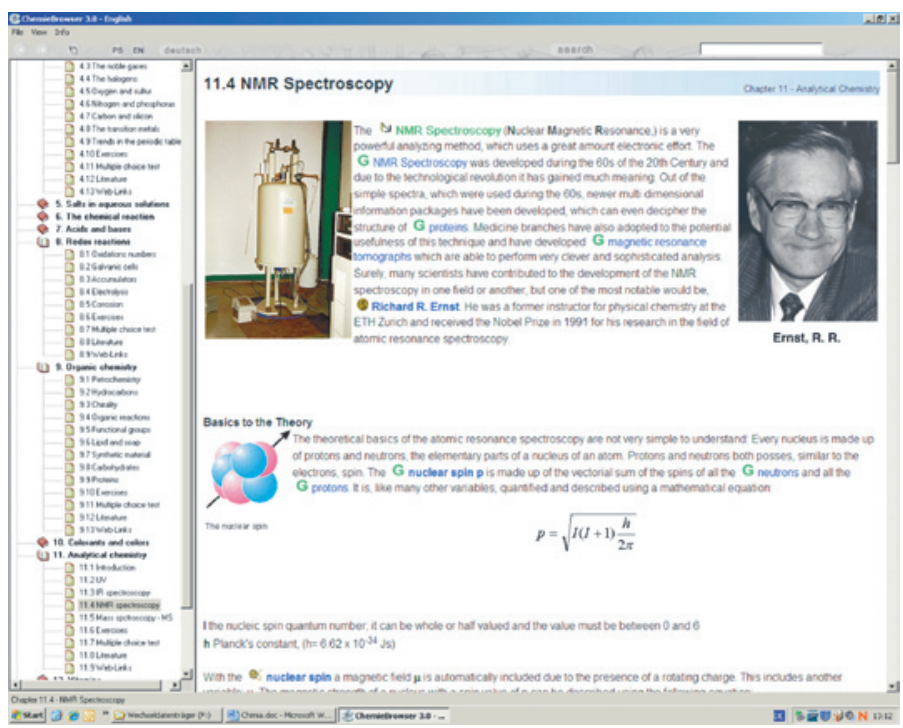

If you teach chemistry in a Swiss school or if you are involved with chemistry teaching at school level and would like to contribute to this column (a chemistry experiment, a project, etc.) please contact Dr. Roland Kunz: E-mail: kunz@oci.uzh.ch 